\title{
DNA methyltransferase 3a modulates chemosensitivity to gemcitabine and oxaliplatin via CHK1 and AKT in p53-deficient pancreatic cancer cells
}

\author{
WEI JING ${ }^{1,2}$, NA SONG $^{3}$, YUNPENG LIU ${ }^{3}$, XIUJUAN QU ${ }^{3}$, KEZUO HOU $^{3}$, \\ XIANGHONG YANG ${ }^{2}$ and XIAOFANG $\mathrm{CHE}^{3}$
}

\begin{abstract}
Departments of ${ }^{1}$ Oncology and ${ }^{2}$ Pathology, Shengjing Hospital of China Medical University, Shenyang, Liaoning 110004;
${ }^{3}$ Department of Medical Oncology, The First Hospital of China Medical University, Shenyang, Liaoning 110001, P.R. China
\end{abstract}

Received December 17, 2016; Accepted August 1, 2017

DOI: $10.3892 / \mathrm{mmr} .2017 .7923$

\begin{abstract}
The aberrant expression of DNA methyltransferases (DNMTs) has been considered to be associated with pancreatic carcinogenesis and progression. DNMT3a is widely involved in cell proliferation and cell cycle progression in pancreatic ductal adenocarcinoma (PDAC) cells. However, its regulation of chemosensitivity to gemcitabine (GEM) and oxaliplatin (OXA) in p53-deficient PDAC remains unclear. In the present study, the effect and mechanisms of DNMT3a on GEM and OXA chemosensitivity in p53-deficient PDAC cells were investigated using MTT assay, cell cycle analysis, apoptosis analysis and western blotting. The treatment of GEM and OXA induced $\mathrm{S}$ phase arrest by DNA damage, and enhanced the activation of the AKT signaling pathway in Panc-1 cells. Downregulation of DNMT3a increased the chemosensitivity to both GEM and OXA in Panc-1 cells. DNMT3a depletion distinctly abolished $\mathrm{S}$ phase arrest induced by GEM and OXA. Further research demonstrated that activation inhibition of CHK1 and AKT, as well as an increase in apoptosis, were involved in DNMT3a-mediated chemosensitivity to GEM and OXA. Taken together, these data demonstrated that DNMT3a serves a crucial role in the regulation of chemosensitivity to
\end{abstract}

Correspondence to: Professor Xianghong Yang, Department of Pathology, Shengjing Hospital of China Medical University, 36 Sanhao Street, Heping, Shenyang, Liaoning 110004, P.R. China E-mail: xhyang4933@vip.sina.com

Professor Xiaofang Che, Department of Medical Oncology, The First Hospital of China Medical University, 155 North Nanjing Street, Heping, Shenyang, Liaoning 110001, P.R. China

E-mail: xf_che@hotmail.com

Abbreviations: PDAC, pancreatic ductal adenocarcinoma; GEM, gemcitabine; OXA, oxaliplatin; DNMTs, DNA methyltransferases; CHK1, cell cycle checkpoint kinase 1

Key words: cell cycle checkpoint kinase 1, DNA methyltransferase 3a, pancreatic ductal adenocarcinoma, chemosensitivity
GEM and OXA, and suggests a promising therapeutic target for p53-deficient PDAC.

\section{Introduction}

Pancreatic ductal adenocarcinoma (PDAC) with its highly lethal malignancy affected $>79,400$ people in China in 2015 (1). While it is a rapidly invasive, metastatic tumor, nearly $80 \%$ of PDAC patients are unresectable at diagnosis due to advanced stages or distant metastasis. The prognosis of PDAC has not been significantly improved, despite advances in comprehensive treatment (2). The overall 5-year survival rate for PDAC is still $\sim 5 \%$ (1). Comprehensive treatment, particularly chemotherapy, is the main strategy for most PDAC patients. However, the effect of chemotherapy is still limited, for both monotherapy and polytherapy.

A variety of molecular and genetic changes exist in the development of PDAC $(3,4)$. Aberrant DNA hypermethylation is considered to be associated with tumorigenesis in human pancreatic tumors (5). DNA methyltransferases (DNMTs), the key cellular enzymes in epigenetic modifications, serve a major role in transferring the methyl group to cytosine in $\mathrm{CpG}$ islands, and are comprised of the three families of DNMT1, DNMT2, DNMT3a and DNMT3b.

DNMT1 is responsible for the maintenance of the DNA methylation pattern during DNA replication, whereas DNMT3 functions as a de novo methyltransferase acting on unmethylated and hemimethylated DNA. DNMTs restrain tumor suppressor gene transcription by promoting methylation of $\mathrm{CpG}$ islands in the promoter, thus contributing to the occurrence and development of the tumor. Previous studies have demonstrated that DNMT1 and DNMT3a are overexpressed in a variety of human tumors, including gastric cancer and pancreatic cancer $(6,7)$, and overexpression of DNMT1 and DNMT3a is inversely associated with the prognosis of PDAC $(8,9)$. Methylation-mediated tumor suppressor gene silencing, which does not involve altering the DNA base sequence, can be reversed by pharmacological or chemical intervention. Therefore, DNMTs have been considered as potential anti-cancer therapeutic targets (10). Inhibition of DNMT1 had synergic effects on the cytotoxicity induced by 
chemotherapeutic drugs in multiple tumor models, including pancreatic cancer $(11,12)$. However, the role of DNMT3a in chemosensitivity remained elusive in PDAC.

Gemcitabine (GEM) and oxaliplatin (OXA) are DNA damage agents, which have been applied in the treatment of PDAC extensively. GEM and 5-fluorouracil (5-FU) have been used as the main chemotherapeutic regimens for PDAC in the last two decades. Recently, clinical studies demonstrated that combined GEM and erlotinib or albumin-bound paclitaxel therapy improved overall survival by $<2$ months in metastatic PDAC $(13,14)$. Furthermore, another combined chemotherapeutic regimen of FOLFIRINOX, including OXA, irinotecan, 5-FU and leucovorin, improved the median overall survival for 4.3 months compared with GEM monotherapy as a first-line therapy for patients with metastatic PDAC (15). Therefore, the objective response of GEM and OXA still remains limited (16). Thus, there is an urgent need to improve chemotherapeutic efficacy in PDAC.

DNA damage in cancer cells caused by DNA damage agents raises the activation of cellular responses, including p53 and serine-protein kinase ATM-cell cycle checkpoint kinase $(\mathrm{CHK}) 2$ and serine/threonine-protein kinase ATR (ATR)-CHK1 pathways, which cause the DNA damage response. It induces cell cycle arrest to repair DNA damage, evading the cytotoxicity of chemotherapeutic agents. P53-deficient cancer cells, unlike normal cells, rely mainly on phosphorylation of S or G2 CHK1, which induces S phase arrest in response to DNA damage, instead of p53 (17). An accumulation of phosphorylated CHK1 induced by GEM treatment leads to S-phase arrest in Panc-1 cells, which prevents premature mitotic entry, and CHK1 depletion enhances GEM-mediated cytotoxicity and radiosensitization (18). Inhibition of CHK1 potentiates the cytotoxicity of irinotecan in triple-negative breast cancer (19), and overcomes the cisplatin resistance in head and neck cancer cells with loss of functional p53 (20). Therefore, CHK1 is regarded as a potential target in p53-deficient cancer, such as PDAC, with nearly $50 \%$ patients being p53-deficient. A previous study demonstrated DNMT3a mediates the cell cycle progression in PDAC cells. However, whether DNMTs affect the activation of CHK1 is unknown.

Previous research demonstrated DNMT1 and DNMT3a are widely expressed in PDAC, mediating the proliferation and cell cycle progression in PDAC cells. However, in our previous work, it was found that downregulation of DNMT3a had synergic effects with GEM or OXA in p53-deficient PDAC cells, which was not detected in DNMT1 inhibition (data not published). The present study investigated the regulation of DNMT3a on chemosensitivity to GEM and OXA, and the potential mechanisms in p53-deficient PDAC cells. Additionally, the role of DNMT3a on CHK1 activity, which contributes to GEM and OXA sensitivity, was assessed.

\section{Materials and methods}

Cell culture and reagents. The Panc-1 p53-deficient pancreatic cancer cells were obtained from the Type Culture Collection of the Chinese Academy of Sciences (Shanghai, China). Panc-1 cells were cultured in RPMI1640 medium (Gibco; Thermo Fisher Scientific, Inc., Waltham, MA, USA) supplemented with
$10 \%$ fetal bovine serum (FBS), and $100 \mathrm{U} / \mathrm{ml}$ penicillin-streptomycin at $37^{\circ} \mathrm{C}$ and $5 \% \mathrm{CO}_{2}$. GEM was obtained from Eli Lilly, Inc. (Indianapolis, IN, USA). Oxaliplatin was purchased from Sanofi-Aventis, Inc. (Paris, France). Antibodies against CHK1 (cat. no. 2360S), phosphorylated (p)-CHK1 (Ser345; cat. no. 2348S), poly[ADP-ribose] polymerase (PARP; cat. no. 9542L), protein kinase B (AKT; cat. no. 9272S), p-AKT (Ser473; cat. no. 9271L), p-extracellular signal-regulated kinase (ERK)1/2 (Thr202/Tyr204; cat. no. 4370S), Caspase-3 (cat. no. 9663S) and $\gamma$-histone H2AX ( $\gamma$-H2AX; cat. no. 9718S) were obtained from Cell Signaling Technology, Inc. (Danvers, MA, USA). Anti-DNMT3a (cat. no. sc-20703), anti-GAPDH (cat. no. sc-25778), anti-ERK (cat. no. sc-514302), and secondary goat anti-rabbit (cat. no. sc-2007) and goat anti-mouse antibodies (cat. no. sc-2039) were obtained from Santa Cruz Biotechnology, Inc. (Dallas, TX, USA).

Transient transfection. Small interfering RNA (siRNA) of DNMT3a from Shanghai Gemma Pharmaceutical Technology, Co., Ltd. (Shanghai, China) was used: 5'-GCGUCACACAGA AGCAUAUTtAUAUGCUUCUGUGUGACGCTT-3'. The negative-control siRNA sequence was 5'-AATTCTCCGAAC GTGTCACGT-3'. Panc-1 cells were transfected with DNMT3a siRNA, negative-control siRNA and Lipofectamine 2000 (Invitrogen; Thermo Fisher Scientific, Inc.) according to the manufacturer's protocol, and the transfection medium was replaced 4-6 $\mathrm{h}$ after transfection.

MTT assay. Panc-1 cells were seeded at $6 \times 10^{3}$ cells per well into 96-well plates with three replicate wells for each condition. Cells treated with GEM and OXA were cultured in 96-well plates for $48 \mathrm{~h}$. A total of $24 \mathrm{~h}$ after transfection, GEM and OXA were added to each corresponding well to continue culturing for $48 \mathrm{~h}$, with final concentration of 1 and $5 \mu \mathrm{M}$, respectively. Cells were then harvested for MTT assay. MTT $(20 \mu \mathrm{l} ; 5 \mathrm{mg} / \mathrm{ml})$ reagent was added to each well, and the incubation continued for $4 \mathrm{~h}$ at $37^{\circ} \mathrm{C}$. After removal of the supernatant, dimethyl sulfoxide (DMSO; $200 \mu \mathrm{l} /$ well) was added to dissolve the formazan crystals. The optical density was measured at $570 \mathrm{~nm}$ with a microplate reader (Model 550, Bio-Rad Laboratories, Inc., Hercules, CA, USA). All experiments were repeated three times.

Western blot analysis. The cells were washed with ice-cold PBS twice, lysed in lysis buffer (1\% Triton X-100, $50 \mathrm{mM}$ Tris- $\mathrm{HCl} \mathrm{Ph}$ 7.4, $150 \mathrm{mM} \mathrm{NaCl}, 10 \mathrm{mM}$ EDTA, $100 \mathrm{mM}$ $\mathrm{NaF}, 1 \mathrm{mM} \mathrm{Na} \mathrm{VO}_{4}, 1 \mathrm{mM}$ PMSF and $2 \mu \mathrm{g} / \mathrm{ml}$ aprotinin) and quantified with the Coomassie brilliant blue G-250 method (Shanghai Maikun Chemical Co., Ltd, Shanghai, China). The supernatant was diluted in $3 \mathrm{X}$ SDS loading buffer and then boiled for $5 \mathrm{~min}$. Proteins (20 $\mu \mathrm{g}$ ) were separated on $8-12 \%$ gels by SDS-PAGE, then transferred onto a polyvinylidene difluoride membrane. The membranes were blocked with 5\% skimmed milk in TBS with Tween 20 (TBST) buffer $(10 \mathrm{mM}$ Tris-HCl pH 7.4, $150 \mathrm{mM} \mathrm{NaCl}, 0.1 \%$ Tween 20) at room temperature for $1 \mathrm{~h}$. The membranes were incubated overnight with the antibodies CHK1 (1:1,000), p-CHK1 (1:500), PARP $(1: 1,000), \operatorname{AKT}(1: 1,000)$, p-AKT $(1: 500)$, p-ERK1/2 (1:500), Caspase-3 (1:1,000), $\gamma$-H2AX $(1: 1,000)$, DNMT3a $(1: 1,000)$, GAPDH $(1: 1,000)$ and ERK1/2 $(1: 500)$ at $4^{\circ} \mathrm{C}$. Following 
A

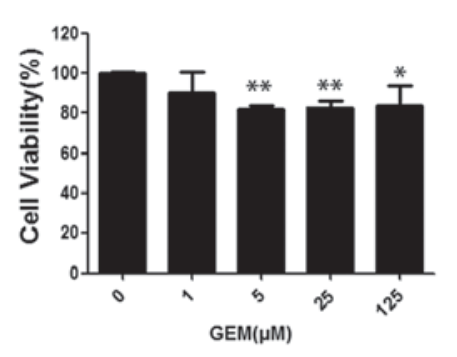

B

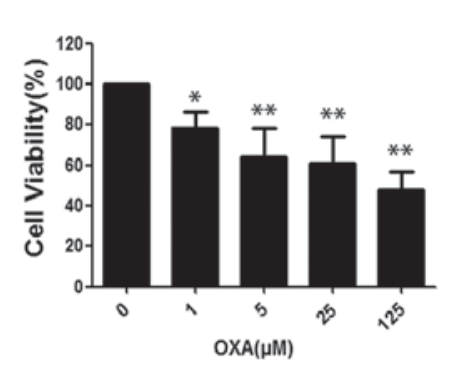

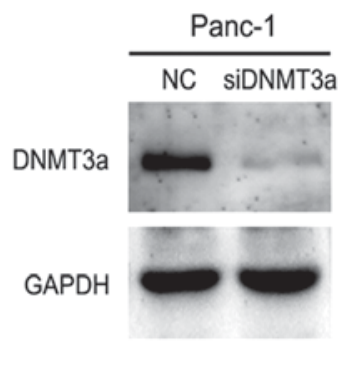

C
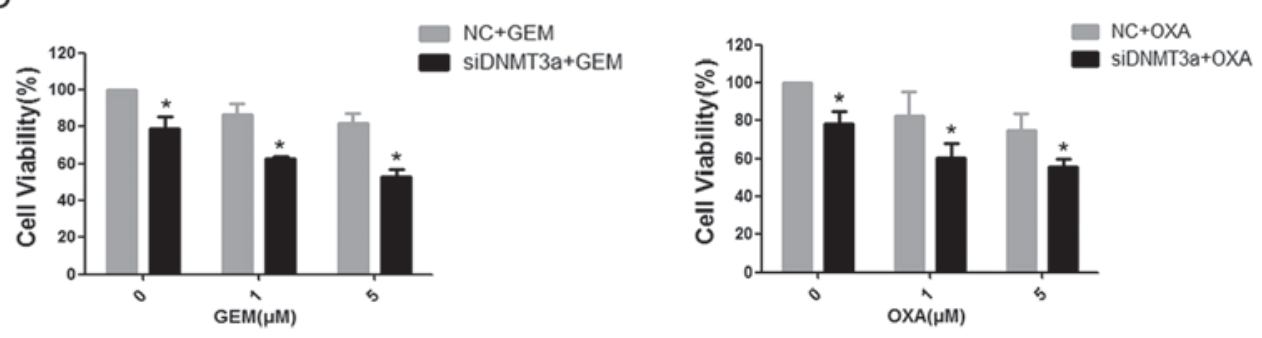

Figure 1. DNMT3a downregulation leads to a chemosensitivity increase to GEM and OXA treatment in Panc-1 cells. (A) MTT assay demonstrated the cell viability rates of Panc-1 cells treated by GEM and OXA for $48 \mathrm{~h}$. ${ }^{*} \mathrm{P}<0.05,{ }^{* *} \mathrm{P}<0.01$ vs. $0 \mu \mathrm{M}$ OXA and GEM. (B) Knockdown of DNMT3a protein was confirmed in Panc-1 cells by western blot analysis. (C) Knockdown of DNMT3a with siRNA led to increased sensitivity towards GEM and OXA, as measured by MTT assay. "P<0.05 vs. NC + GEM/OXA treatment. Data are presented as the mean \pm standard deviation of three independent experiments. GEM, gemcitabine; si, small interfering; NC, negative control; DNMT3a, DNA methyltransferase 3a; OXA, oxaliplatin.

washing with TTBS buffer three times, the membrane was incubated with secondary goat anti-rabbit $(1: 1,000)$ and goat anti-mouse (1:500) antibodies for $30 \mathrm{~min}$ at room temperature. Finally, the protein bands were ultimately visualized with a MicroChemi 4.2 Gel Capture version 6.12 (DNR Bio-Imaging Systems, Ltd., Neve Yamin, Israel).

Cell cycle analysis. Panc-1 cells were treated with GEM $(5 \mu \mathrm{M})$ and OXA $(5 \mu \mathrm{M})$ for 12 and $24 \mathrm{~h}$, respectively. In the meantime, the cells were transfected with negative-control siRNA or DNMT3a siRNA for $48 \mathrm{~h}$. After transfection, GEM was added for $12 \mathrm{~h}$ with a final concentration of $5 \mu \mathrm{M}$, and OXA was added for $24 \mathrm{~h}$ with a final concentration of $5 \mu \mathrm{M}$. Cells were washed with cold PBS twice, and fixed with ice-cold $70 \%$ ethanol overnight at $4^{\circ} \mathrm{C}$ and then incubated with $100 \mu \mathrm{g} / \mathrm{ml}$ RNase A in PBS for $30 \mathrm{~min}$ at $37^{\circ} \mathrm{C}$. Subsequently, cells were stained with propidium iodide (PI; $5 \mathrm{mg} / \mathrm{ml}$ ) for another $30 \mathrm{~min}$ at $37^{\circ} \mathrm{C}$ away from light. A BD Accuri C6 FACScanflow cytometer (BD Biosciences, Franklin Lakes, NJ, USA) and ModFit LT software version 3.3 (modfit-lt. software.informer.com/download; Verity Software House, Inc., Topsham, ME, USA) were used to analyze cell cycle distribution. All experiments were repeated three times.

Apoptosis analysis. Panc-1 cells were seeded at $3 \times 10^{5}$ cells per well into 6-well plates. Cells were transfected with negative-control siRNA, DNMT3a siRNA using Lipofectamine 2000 (Invitrogen; Thermo Fisher Scientific, Inc.) according to the manufacturer's protocol. A total of $24 \mathrm{~h}$ after transfection, GEM and OXA were added for $48 \mathrm{~h}$ with a final concentration of $5 \mu \mathrm{M}$. Subsequently, cells were harvested and resuspended in binding buffer containing Annexin V-FITC and PI according to the instructions of the Annexin V-FITC/PI Apoptosis Detection kit (Invitrogen; Thermo Fisher Scientific, Inc.). The percentage of apoptosis was analyzed by flow cytometry. For each group, the process was repeated three times.

Statistical analysis. All experiments were repeated at least three times. All values are expressed as the mean \pm standard error. Differences between the multiple groups were evaluated by one-way analysis of variance with a post-hoc LSD test, and differences between the two groups were evaluated by Student's t-test (two-tailed). SPSS software version 17.0 (SPSS Inc., Chicago, IL, USA) was used for statistical analysis. $\mathrm{P}<0.05$ was considered to indicate a statistically significant difference.

\section{Results}

DNMT3a downregulation increases GEM and OXA sensitivity of p53-deficient PDAC cells. First, GEM and OXA sensitivity was examined in Panc-1 cells. The results demonstrated that cell viability was inhibited by GEM and OXA in a dose-dependent manner in $48 \mathrm{~h}$. Panc-1 cells treated with GEM had significantly greater viability than cells treated with OXA (Fig. 1A). The cell viability rates of Panc-1 cells treated with $5 \mu \mathrm{M}$ GEM for $48 \mathrm{~h}$ was $82.13 \pm 1.93 \%,(\mathrm{P}=0.007)$, and for cells treated with $5 \mu \mathrm{M}$ OXA was $63.84 \pm 14.49 \%(\mathrm{P}=0.001)$. To identify the role of DNMT3a in regulating chemosensitivity, siRNA was used to knockdown the expression of DNMT3a in Panc-1 cells, and the efficiency of DNMT3a suppression by siRNA was confirmed by western blotting (Fig. 1B). Subsequently, the effects of DNMT3a downregulation on the sensitivity of pancreatic cancer cells to GEM and OXA was examined by MTT assay. The results demonstrated that the downregulation of DNMT3a increased the drug-sensitivity of Panc-1 to both GEM and OXA (Fig. 1C; Table I). These data suggested that DNMT3a downregulation increased sensitivity of Panc- 1 cells to GEM and OXA. 
GEM and OXA induce the activation of AKT and CHK1 in Panc-1 cells. To investigate the effect of GEM and OXA on cell proliferation and DNA damage, western blot analysis and flow cytometry were performed to identify the expression of associated proteins and the cell cycle. p-AKT and p-ERK were gradually elevated by GEM and OXA in Panc-1 cells, which reached a peak at $6 \mathrm{~h}$ following GEM or OXA treatment (Fig. 2A). Flow cytometry revealed that the accumulation in $\mathrm{S}$ phase cells was increased with following treatment of GEM or OXA for 12 and $24 \mathrm{~h}$, respectively (Fig. 2B). The percentage of S phase cells increased from $39.19 \pm 5.43$ to $49.27 \pm 3.99 \%$ following GEM treatment, and $41.48 \pm 2.43$ to $51.81 \pm 3.28 \%$ following OXA treatment (Fig. 2C). Furthermore, $\gamma$-H2AX was activated in a time-dependent manner in Panc-1 cells treated with GEM and OXA. In addition, a significant phosphorylation of CHK1 was observed after drug treatment for $6 \mathrm{~h}$ (Fig. 2D). Taken together, these data suggested that GEM and OXA, as DNA damage agents, not only induced cell cycle arrest, but also stimulated the cell proliferation signal pathway, which may cause limited inhibition of GEM and OXA in Panc-1 cells.

DNMT3a downregulation promotes cell apoptosis induced by GEM and OXA. The cytotoxicity of GEM and OXA not only inhibited proliferative signal and cell cycle arrest, but also induced apoptosis in cancer cells. Annexin V-FITC/PI and western blot analysis were performed to elucidate cell apoptosis. Annexin V-FITC/PI demonstrated that DNMT3a downregulation combined with GEM or OXA increased cell apoptosis (GEM + NC vs. GEM + siDNMT3a: $7.97 \pm 2.11 \%$ vs. $19.87 \pm 3.23 \%, \mathrm{P}<0.001 ;$ OXA $+\mathrm{NC}$ vs. OXA + siDNMT3a: $4.4 \pm 1.65 \%$ vs. $16.57 \pm 2.44 \%, \mathrm{P}<0.001$; Fig. $3 \mathrm{~A}$ and $\mathrm{B})$. Similarly, dual inhibition with DNMT3a siRNA and drug treatment distinctly induced cleavage of PARP and caspase-3 compared to single agent treatment (Fig. 3C). Thus, DNMT3a downregulation increased apoptosis in GEM and OXA-treated Panc-1 cells.

DNMT3a downregulation abrogates the activation of AKT and CHK1 and cell cycle arrest induced by GEM and OXA. Next, the change of AKT and ERK signaling and cell cycle when DNMT3a was downregulated was investigated. The results demonstrated that downregulation of DNMT3a could significantly inhibit the AKT activation induced by GEM and OXA at $6 \mathrm{~h}$, while no obvious change of p-ERK was observed in Panc-1 cells (Fig. 4A). Additionally, downregulation of DNMT3a distinctly abolished the blockage of $S$ phase arrest induced by GEM and OXA at 12 and $24 \mathrm{~h}$, and enhanced $\mathrm{G}_{0} / \mathrm{G}_{1}$ phase arrest (Fig. 4B). Downregulation of DNMT3a restored the $\mathrm{S}$ phase arrest response to GEM and OXA in Panc-1 cells $($ GEM + NC vs. GEM + siDNMT3a: $35.49 \pm 6.37 \%$ vs. $17.88 \pm 4.25 \%, \mathrm{P}=0.001 ;$ OXA + NC vs. OXA + siDNMT3a: $45.35 \pm 6.15 \%$ vs. $24.18 \pm 4.93 \%, P<0.001$ ), and raised $\mathrm{G}_{0} / \mathrm{G}_{1}$ percentage (GEM + NC vs. GEM + siDNMT3a: $64.51 \pm 6.37 \%$ vs. $82.12 \pm 4.25 \%, \mathrm{P}=0.003$; OXA $+\mathrm{NC}$ vs. OXA + siDNMT3a: $46.35 \pm 6.91 \%$ vs. $75.26 \pm 4.00 \%, \mathrm{P}<0.001$; Fig. $4 \mathrm{C}$ ) Furthermore, $\mathrm{p}$-CHK1 expression induced by GEM and OXA at $6 \mathrm{~h}$ apparently decreased after DNMT3a downregulation in Panc-1 cells (Fig. 4D). These results indicated that DNMT3a downregulation enhanced the sensitivity of GEM and OXA in
Table I. Effect of DNMT3a downregulation on sensitivity to GEM and OXA in Panc-1 cells.

\begin{tabular}{llll}
\hline & \multicolumn{3}{c}{ Cell viability (\%) } \\
\cline { 2 - 4 } Concentration $(\mu \mathrm{M})$ & $\mathrm{NC}$ & siDNMT3a & P-value \\
\hline GEM & & & \\
0 & $100.0 \pm 0.00$ & $79.26 \pm 6.14$ & 0.028 \\
1 & $86.54 \pm 6.21$ & $62.54 \pm 1.31$ & 0.016 \\
5 & $81.96 \pm 5.29$ & $52.98 \pm 3.85$ & 0.012 \\
OXA & & & \\
0 & $100.0 \pm 0.00$ & $78.32 \pm 6.65$ & 0.029 \\
1 & $82.64 \pm 12.42$ & $60.67 \pm 7.73$ & 0.028 \\
5 & $74.59 \pm 9.39$ & $55.85 \pm 4.23$ & 0.025 \\
\hline
\end{tabular}

GEM, gemcitabine; si, small interfering; NC, negative control; DNMT3a, DNA methyltransferase 3a; OXA, oxaliplatin.

Panc-1 cells by disrupting the activation of AKT, CHK1 and $S$ phase arrest.

\section{Discussion}

Aberrant methylation has been considered to be involved in pancreatic carcinogenesis and progression. Inhibition of the function of DNMTs has been proven to be a potential target for improving survival and reinforcing therapeutic effect $(5,9,10)$. The present study reported that the cytotoxicity of GEM and OXA was significantly enhanced in DNMT3a knockdown Panc-1 cells. The underlying mechanisms suggested DNMT3a downregulation inhibited the activation of CHK1 and decreased the $\mathrm{S}$ phase fraction in Panc-1 cells after drug administration. On the other hand, DNMT3a downregulation also suppressed AKT activation to inhibit the responsiveness to DNA damage, and increase cell apoptosis caused by chemotherapeutic drugs. These data suggested that DNMT3a served an important role in the chemotherapy sensitivity of p53-deficient PDAC cells.

CHK1 is the most important serine/threonine kinase in the cell cycle checkpoint during DNA damage responses. It is overexpressed in a variety of human tumors, especially in breast, cervical and gastric carcinomas (21-23). The activation of the ATR-CHK1 pathway in response to DNA damage leads to cell cycle arrest for DNA repair during the application of radiotherapy or anti-cancer therapy agents (24). Accordingly, CHK1 inhibition potentiates the sensitivity of multiple DNA damage chemotherapy agents by restraining the DNA damage response, especially antimetabolites, notably GEM, which is widely used in various of solid tumors $(25,26)$. In addition, decreased expression of CHK1 leads to sensitization of mesothelioma cells to platinum, and hepatocellular carcinoma cells to cisplatin $(27,28)$. In p53-deficient cells, CHK1 dominates in cell cycle regulation after DNA damage instead of $\mathrm{G}_{1} / \mathrm{G}_{0}$ checkpoint $\mathrm{p} 53$. Therefore, p53-deficient cancer cells are considered to be more sensitive to therapeutic strategies that combine DNA damaging agents with CHK1 inhibitors (29). Encouraging 

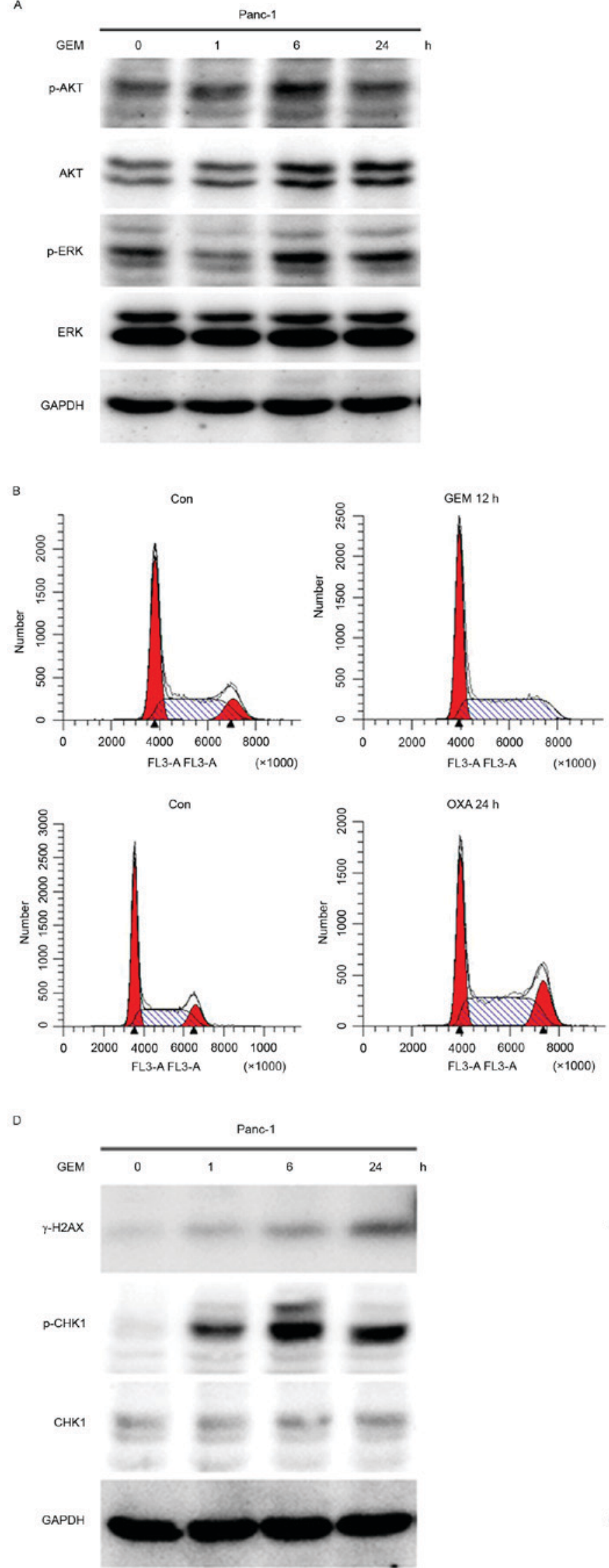
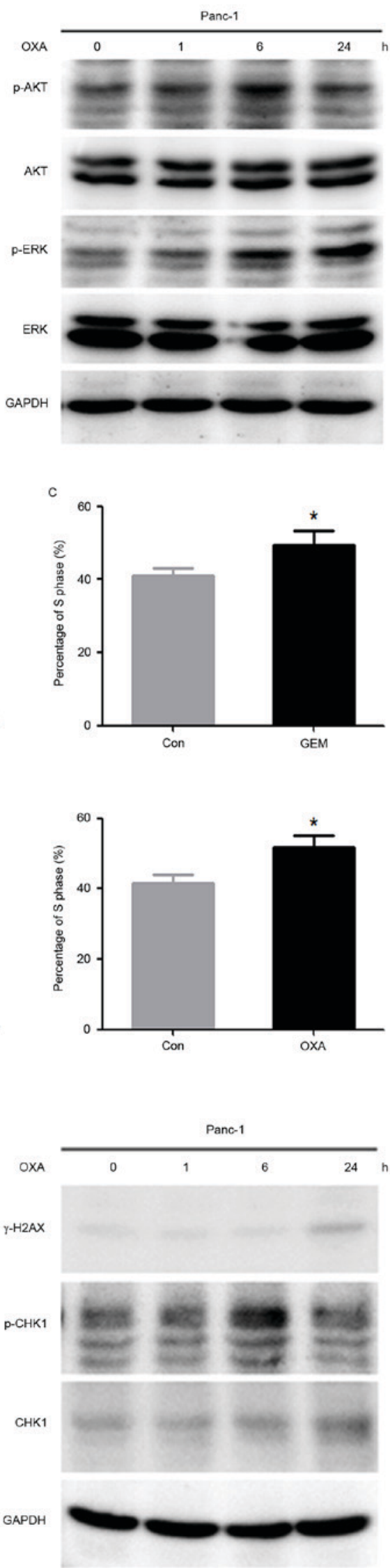

Figure 2. GEM and OXA induces activation of AKT and CHK1 in Panc-1 cells. (A) The expression of p-AKT, AKT, p-ERK and ERK in Panc-1 cells treated with GEM $(5 \mu \mathrm{M})$ and OXA $(5 \mu \mathrm{M})$ for $0,1,6,24 \mathrm{~h}$ was analyzed by western blot. (B) The cell cycle was detected by flow cytometry in Panc-1 cells after GEM $(5 \mu \mathrm{M})$ and OXA $(5 \mu \mathrm{M})$ treatment for 12 and $24 \mathrm{~h}$ and $(\mathrm{C})$ percentage of cells in S phase. (D) Cells were treated with GEM $(5 \mu \mathrm{M})$ and OXA $(5 \mu \mathrm{M})$ for $0,1,6$ and $24 \mathrm{~h}$. Expression of $\gamma-\mathrm{H} 2 \mathrm{AX}, \mathrm{p}-\mathrm{CHK} 1$ and $\mathrm{CHK} 1$ were determined by western blot analysis. Data are presented as the mean \pm standard deviation. "P<0.05. Con, control; GEM, gemcitabine; OXA, oxaliplatin; p, phosphorylated; AKT, protein kinase B; $\gamma$-H2AX, $\gamma$-histone H2AX; ERK, extracellular signal-regulated kinase; CHK1, cell cycle checkpoint kinase 1. 
A NC

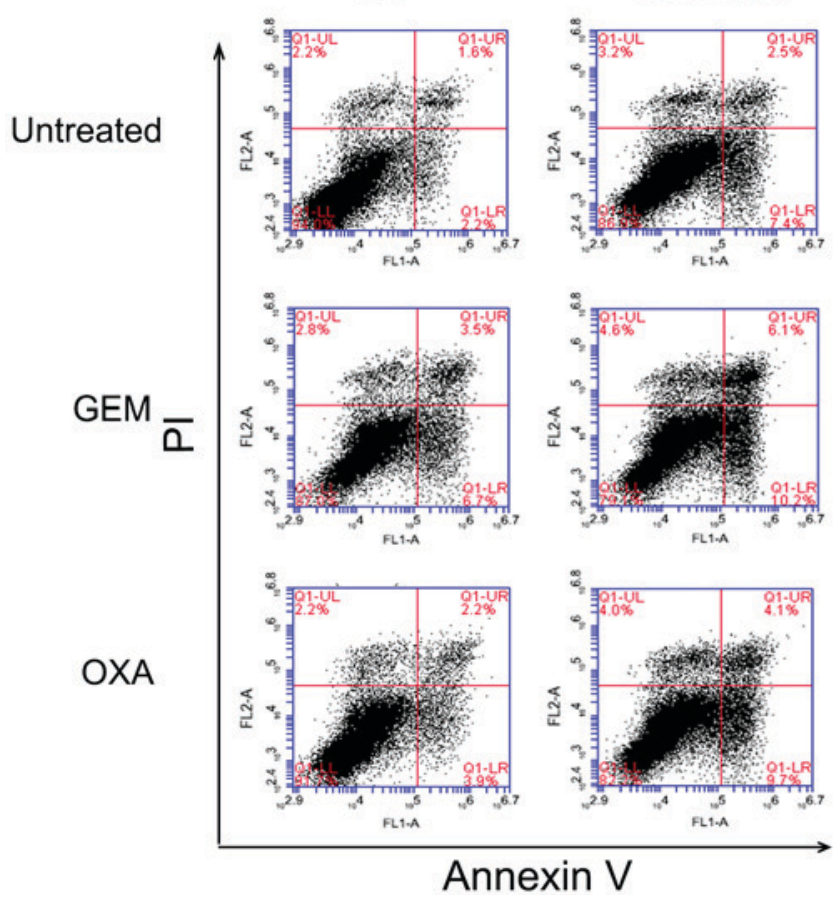

B
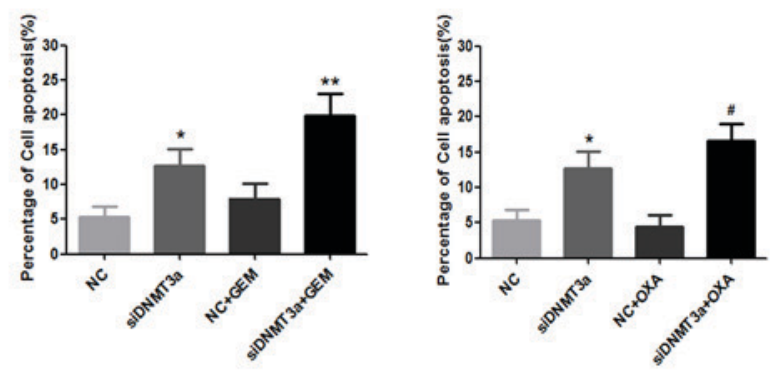

C

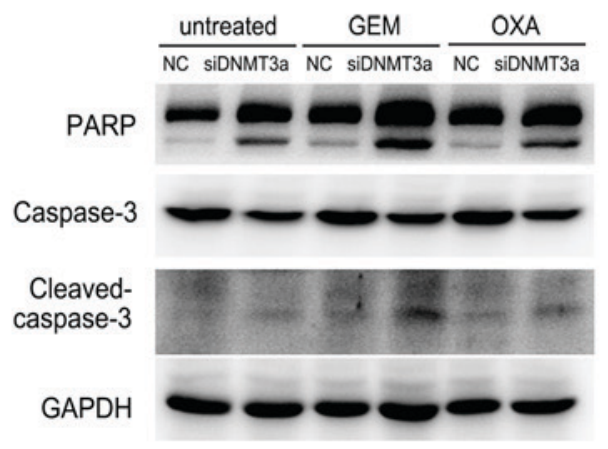

Figure 3. Effect of a combination of DNMT3a downregulation with GEM and OXA treatment on apoptosis. (A) After transfection with DNMT3a siRNA for $72 \mathrm{~h}$ and treatment with GEM $(5 \mu \mathrm{M})$ and OXA $(5 \mu \mathrm{M})$ for $48 \mathrm{~h}$, Annexin V-FITC/PI was applied to detect cell apoptosis. (B) The percentage of apoptotic cells (Annexin V-positive cells). (C) Panc-1 cells were transfected with DNMT3a siRNA, followed by treatment of GEM (5 $\mu \mathrm{M}$ ) or OXA (5 $\mu \mathrm{M})$ for $48 \mathrm{~h}$, and expression of the apoptosis-associated proteins PARP and caspase-3 were determined by western blot analysis. Data are presented as the mean \pm standard deviation. ${ }^{*} \mathrm{P}<0.01$ vs. NC, ${ }^{* *} \mathrm{P}<0.001$ vs. $\mathrm{NC}+\mathrm{GEM},{ }^{\#} \mathrm{P}<0.001$ vs. NC + OXA. GEM, gemcitabine; OXA, oxaliplatin; NC, negative control; si, small interfering; DNMT3a, DNA methyltransferase 3a; FITC, fluorescein isothiocyanate; PI, propidium iodide; PARP, poly[ADP-ribose] polymerase.

results were obtained with combination of demethylating agents and classic anticancer chemotherapeutics in colorectal cancer. DNMTs inhibitors could potentiate the inhibitory effects of OXA in colorectal cancer cells, while activation of CHK1 respond to DNA damage response varied in different DNMT inhibitors (30). The role executed by different members of the DNMT family is still unidentified and needs to be evaluated (30). In the present study, it was demonstrated that $\mathrm{p}-\mathrm{CHK} 1$ and $\gamma-\mathrm{H} 2 \mathrm{AX}$ expression levels were elevated in p53-deficient Panc-1 cells following DNA damage caused by GEM and OXA, accompanied by cell accumulation in $\mathrm{S}$ phase. Inhibition of DNMT3a restored the $\mathrm{S}$ phase fraction and CHK1 activation, arrested cells in $\mathrm{G}_{0} / \mathrm{G}_{1}$ phase and increased response to chemotherapy treatment. These results highlighted that DNMT3a downregulation enhanced the sensitivity of GEM and OXA in p53-deficient Panc-1 cells by disrupting the activation of $\mathrm{CHK} 1$. However, there was no methylation loci in the promoter of CHK1, and molecules directly regulated by DNMT3a through epigenetic regulation, which could modify the phosphorylation of $\mathrm{CHK} 1$, were not investigated. Thus, DNMT3a may regulate CHK1 activation via an indirect effect in Panc-1 cells, and further study is needed to elucidate the underlying mechanisms.

AKT serves a critical role in regulating cellular processes in cancer cells, including cell proliferation, anti-apoptosis, migration and drug resistance. An excessive activation of p-AKT may induce multidrug resistance in cancer (31). In pancreatic cancer, abnormal AKT activation is an analogous mechanism affecting chemoresistance to GEM. The combination with an AKT inhibitor and GEM synergistically inhibited pancreatic cancer cell growth (32). An excessive activation of AKT made a great contribution to OXA resistance in hepatocellular carcinoma (33). In addition, activation of AKT can also be induced by DNA damage. DNA damage caused by cisplatin induces activation of AKT in platinum-resistant ovarian cancer cells, implicating AKT-activation as a resistance mechanism (34). The present study observed that AKT/ERK-mediated pro-survival signaling was markedly activated following treatment with GEM and OXA in Panc-1 cells. However, downregulation of DNMT3a merely decreased the activation of AKT in response to cytotoxic agents, and improved the sensitivity to GEM and OXA. These results revealed that there was a suppression of the proliferation signal as a synergistic therapeutic effect of GEM/OXA and DNMT3a inhibition.

According to previous studies, chemotherapeutic drugs cause anti-tumor effects partly by promoting cell apoptosis. In the present study, the increased cell apoptosis was detected in cells combined with DNMT3a downregulation and GEM/OXA, which was considered to be induced by the effect of irreversible DNA damage and depressing AKT activation. However, further research is needed to discover whether there are other factors leading to apoptosis.

In conclusion, the present study demonstrated that DNMT3a downregulation enhanced the chemotherapeutic toxicity of GEM and OXA by suppressing the phosphorylation 
A

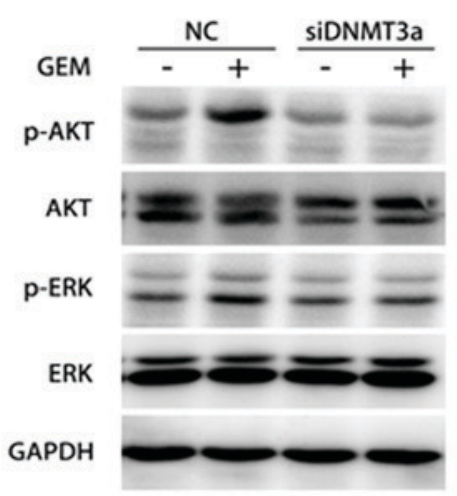

B

NC

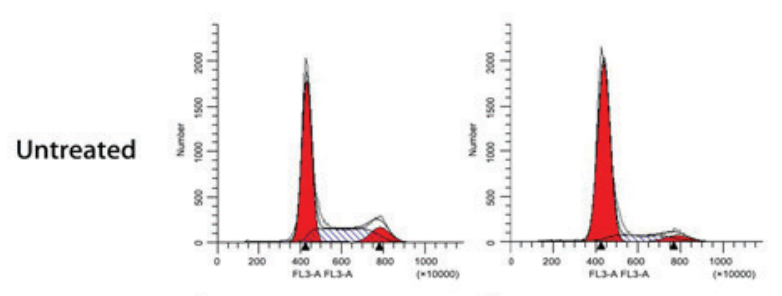

GEM

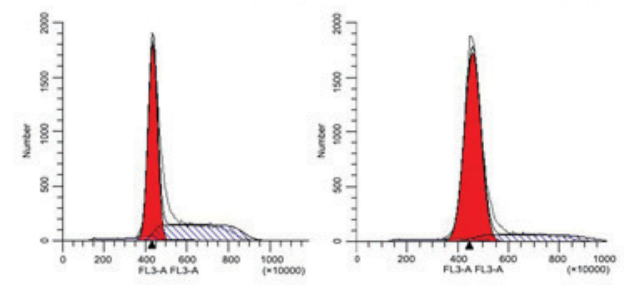

OXA

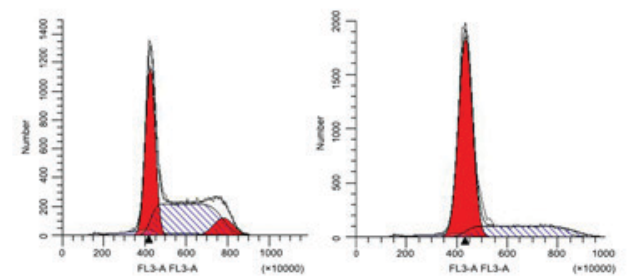

D

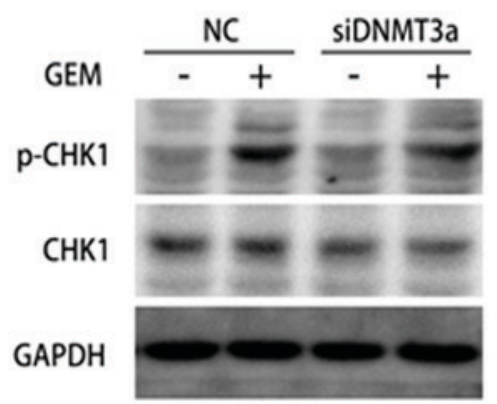

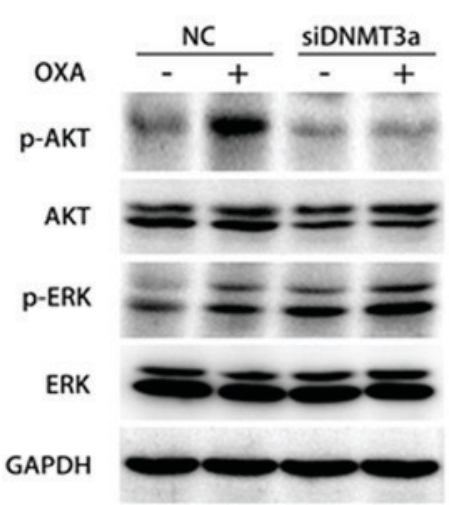

C
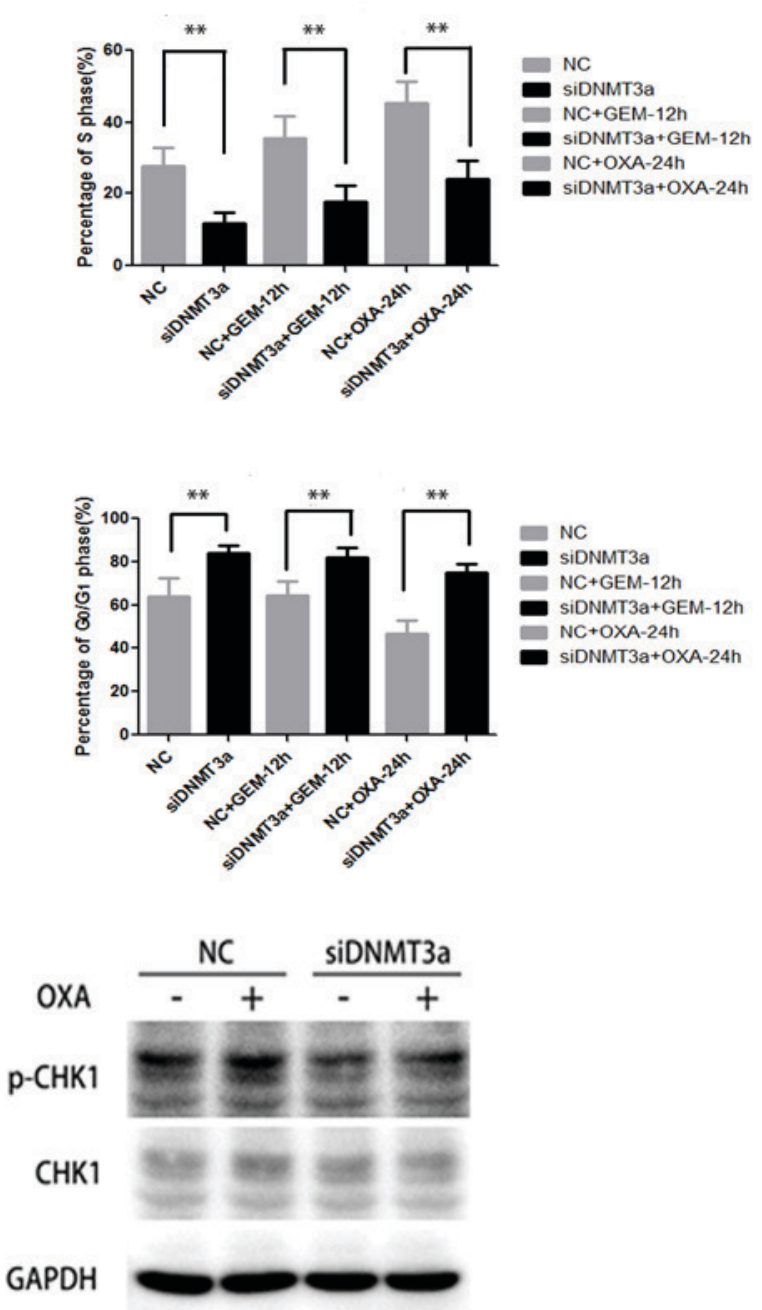

Figure 4. Effect of DNMT3a downregulation by siRNA on activation of AKT, CHK1 and cell cycle arrest in Panc-1 cells. (A) Panc-1 cells were transfected with DNMT3a siRNA, then the cells were treated by GEM $(5 \mu \mathrm{M})$ and OXA $(5 \mu \mathrm{M})$ for $6 \mathrm{~h}$. The expression level of p-AKT, AKT, p-ERK and ERK in negative control and DNMT3a knockdown cells was assessed by western blot analysis. (B) Following transfection with DNMT3a siRNA and treatment by GEM $(5 \mu \mathrm{M})$ and OXA $(5 \mu \mathrm{M})$ for 12 and $24 \mathrm{~h}$, flow cytometry was applied to observe the cell cycle alteration. (C) Percentage of cells in $\mathrm{S}$ and $\mathrm{G}_{0} / \mathrm{G}_{1}$ phases. (D) Western blot analysis of p-CHK1 and CHK1 expression in Panc-1 cells, which were transfected with DNMT3a siRNA, then treated with GEM $(5 \mu \mathrm{M})$ and OXA $(5 \mu \mathrm{M})$ for $6 \mathrm{~h}$. Data are presented as the mean \pm standard deviation. ${ }^{* *} \mathrm{P}<0.01$ vs. corresponding control cells. GEM, gemcitabine; OXA, oxaliplatin; NC, negative control; si, small interfering; DNMT3a, DNA methyltransferase 3a; p, phosphorylated; AKT, protein kinase B; CHK1, cell cycle checkpoint kinase 1; ERK, extracellular signal-regulated kinase.

of CHK1 and AKT, inhibiting S phase arrest and promoting apoptosis in Panc-1 cells, suggesting the suppression of DNMT3a sensitized p53-deficient pancreatic cancer to DNA damage chemotherapeutic agents. Therefore, the present study implicated DNMT3a as a promising crucial therapeutic target for p53-deficient PDAC therapy. 


\section{Acknowledgements}

The present study was supported by Outstanding Scientific Foundation of Shengjing Hospital (grant no. 201210) and the National Natural Science Foundation of China (grant no. 81401938).

\section{References}

1. Chen W, Zheng R, Baade PD, Zhang S, Zeng H, Bray F, Jemal A, Yu XQ and He J: Cancer statistics in China, 2015. CA Cancer J Clin 66: 115-132, 2016.

2. Sinn M, Bahra M, Denecke T, Travis S, Pelzer U and Riess H: Perioperative treatment options in resectable pancreatic cancer-how to improve long-term survival. World J Gastrointest Oncol 8: 248-257, 2016.

3. Delpu Y, Hanoun N, Lulka H, Sicard F, Selves J, Buscail L, Torrisani $\mathrm{J}$ and Cordelier P: Genetic and epigenetic alterations in pancreatic carcinogenesis. Current Genomics 12: 15-24, 2011.

4. Biankin AV, Waddell N, Kassahn KS, Gingras MC, Muthuswamy LB, Johns AL, Miller DK, Wilson PJ, Patch AM, Wu J, et al: Pancreatic cancer genomes reveal aberrations in axon guidance pathway genes. Nature 491: 399-405, 2012.

5. Peng DF, Kanai Y, Sawada M, Ushijima S, Hiraoka N, Kosuge T and Hirohashi S: Increased DNA methyltransferase 1 (DNMT1) protein expression in precancerous conditions and ductal carcinomas of the pancreas. Cancer Sci 96: 403-408, 2005.

6. Yang J, Wei X, Wu Q, Xu Z, Gu D, Jin Y, Shen Y, Huang H, Fan $\mathrm{H}$ and Chen J: Clinical significance of the expression of DNA methyltransferase proteins in gastric cancer. Mol Med Rep 4: 1139-1143, 2011.

7. He S, Wang F, Yang L, Guo C, Wan R, Ke A, Xu L, Hu G, Xu X, Shen $\mathrm{J}$ and Wang X: Expression of DNMT1 and DNMT3a are regulated by GLI1 in human pancreatic cancer. PLoS One 6: e27684, 2011.

8. Zhang JJ, Zhu Y, Zhu Y, Wu JL, Liang WB, Zhu R, Xu ZK, Du Q and Miao Y: Association of increased DNA methyltransferase expression with carcinogenesis and poor prognosis in pancreatic ductal adenocarcinoma. Clin Transl Oncol 14: 116-124, 2012.

9. Gao J, Wang L, Xu J, Zheng J, Man X, Wu H, Jin J, Wang K, Xiao H, Li S and Li Z: Aberrant DNA methyltransferase expression in pancreatic ductal adenocarcinoma development and progression. J Exp Clin Cancer Res 32: 86, 2013.

10. Ghoshal K and Bai S: DNA methyltransferases as targets for cancer therapy. Drugs Today (Barc) 43: 395-422, 2007.

11. Mutze K, Langer R, Schumacher F, Becker K, Ott K, Novotny A, Hapfelmeier A, Höfler $\mathrm{H}$ and Keller G: DNA methyltransferase 1 as a predictive biomarker and potential therapeutic target for chemotherapy in gastric cancer. Eur J Cancer 47: 1817-1825, 2011.

12. Nagaraju GP, Zhu S, Wen J, Farris AB, Adsay VN, Diaz R, Snyder JP, Mamoru S and El-Rayes BF: Novel synthetic curcumin analogues EF31 and UBS109 are potent DNA hypomethylating agents in pancreatic cancer. Cancer Lett 341: 195-203, 2013.

13. Von Hoff DD, Ervin T, Arena FP, Chiorean EG, Infante J, Moore M, Seay T, Tjulandin SA, Ma WW, Saleh MN, et al: Increased survival in pancreatic cancer with nab-paclitaxel plus gemcitabine. N Engl J Med 369: 1691-1703, 2013.

14. Moore MJ, Goldstein D, Hamm J, Figer A, Hecht JR, Gallinger S, Au HJ, Murawa P, Walde D, Wolff RA, et al: Erlotinib plus gemcitabine compared with gemcitabine alone in patients with advanced pancreatic cancer: A phase III trial of the National Cancer Institute of Canada Clinical Trials Group. J Clin Oncol 25: 1960-1966, 2007.

15. Vaccaro V, Sperduti I and Milella M: FOLFIRINOX versus gemcitabine for metastatic pancreatic cancer. N Engl J Med 365: 768-769, 2011.

16. Poplin E, Feng Y, Berlin J, Rothenberg ML, Hochster H, Mitchell E, Alberts S, O'Dwyer P, Haller D, Catalano P, et al: Phase III, randomized study of gemcitabine and oxaliplatin versus gemcitabine (fixed-dose rate infusion) compared with gemcitabine (30-min infusion) in patients with pancreatic carcinoma E6201: A trial of the eastern cooperative oncology group J Clin Oncol 27: 3778-3785, 2009.

17. Chen Z, Xiao Z, Gu WZ, Xue J, Bui MH, Kovar P, Li G, Wang G, Tao ZF, Tong Y, et al: Selective Chk1 inhibitors differentially sensitize p53-deficient cancer cells to cancer therapeutics. Int J Cancer 119: 2784-2794, 2006.
18. Morgan MA, Parsels LA, Parsels JD, Mesiwala AK, Maybaum J and Lawrence TS: Role of checkpoint kinase 1 in preventing premature mitosis in response to gemcitabine. Cancer Res 65 : 6835-6842, 2005.

19. Ma CX, Cai S, Li S, Ryan CE, Guo Z, Schaiff WT, Lin L, Hoog J, Goiffon RJ, Prat A, et al: Targeting Chk1 in p53-deficient triple-negative breast cancer is therapeutically beneficial in human-in-mouse tumor models. J Clin Invest 122: 1541-1552, 2012.

20. Gadhikar MA, Sciuto MR, Alves MV, Pickering CR, Osman AA, Neskey DM, Zhao M, Fitzgerald AL, Myers JN and Frederick MJ: Chk1/2 inhibition overcomes the cisplatin resistance of head and neck cancer cells secondary to the loss of functional p53. Mol Cancer Ther 12: 1860-1873, 2013.

21. Albiges L, Goubar A, Scott V, Vicier C, Lefèbvre C, Alsafadi S, Commo F, Saghatchian M, Lazar V, Dessen P, et al: Chk1 as a new therapeutic target in triple-negative breast cancer. Breast 23: 250-258, 2014.

22. Bargiela-Iparraguirre J, Prado-Marchal L, Fernandez-Fuente M, Gutierrez-González A, Moreno-Rubio J, Muñoz-Fernandez M, Sereno M, Sanchez-Prieto R, Perona R and Sanchez-Perez I: CHK1 expression in gastric cancer is modulated by p53 and RB1/E2F1: Implications in chemo/radiotherapy response. Sci Rep 6: 21519, 2016.

23. Xu J, Li Y, Wang F, Wang X, Cheng B, Ye F, Xie X, Zhou C and Lu W: Suppressed miR-424 expression via upregulation of target gene Chk1 contributes to the progression of cervical cancer. Oncogene 32: 976-987, 2013.

24. Ben-Yehoyada M, Wang LC, Kozekov ID, Rizzo CJ, Gottesman ME and Gautier J: Checkpoint signaling from a single DNA interstrand crosslink. Mol Cell 35: 704-715, 2009.

25. Xiao Y, Ramiscal J, Kowanetz K, Del Nagro C, Malek S, Evangelista M, Blackwood E, Jackson PK and O'Brien T: Identification of preferred chemotherapeutics for combining with a CHK1 inhibitor. Mol Cancer Ther 12: 2285-2295, 2013.

26. Barnard D, Diaz HB, Burke T, Donoho G, Beckmann R, Jones B, Barda D, King C and Marshall M: LY2603618, a selective CHK1 inhibitor, enhances the anti-tumor effect of gemcitabine in xenograft tumor models. Invest New Drugs 34: 49-60, 2016.

27. Røe OD, Szulkin A, Anderssen E, Flatberg A, Sandeck H, Amundsen T, Erlandsen SE, Dobra K and Sundstrøm SH: Molecular resistance fingerprint of pemetrexed and platinum in a long-term survivor of mesothelioma. PLoS One 7: e40521, 2012.

28. Hong J, Hu K, Yuan Y, Sang Y, Bu Q, Chen G, Yang L, Li B, Huang P, Chen D, et al: CHK1 targets spleen tyrosine kinase (L) for proteolysis in hepatocellular carcinoma. J Clin Invest 122: 2165-2175, 2012.

29. Koniaras K, Cuddihy AR, Christopoulos H, Hogg A and O'Connell MJ: Inhibition of Chk1-dependent G2 DNA damage checkpoint radiosensitizes p53 mutant human cells. Oncogene 20: 7453-7463, 2001.

30. Flis S, Gnyszka A and Flis K: DNA methyltransferase inhibitors improve the effect of chemotherapeutic agents in SW48 and HT-29 colorectal cancer cells. PLoS One 9: e92305, 2014.

31. Radisavljevic Z: AKT as locus of cancer multidrug resistance and fragility. J Cell Physiol 228: 671-674, 2013.

32. Kim R, Yamauchi T, Husain K, Sebti S and Malafa M: Triciribine phosphate monohydrate, an AKT inhibitor, enhances gemcitabine activity in pancreatic cancer cells. Anticancer Res 35: 4599-4604, 2015.

33. Xiu P, Dong X, Dong X, Xu Z, Zhu H, Liu F, Wei Z, Zhai B, Kanwar JR, Jiang H, et al: Secretory clusterin contributes to oxaliplatin resistance by activating Akt pathway in hepatocellular carcinoma. Cancer Sci 104: 375-382, 2013.

34. Stronach EA, Chen M, Maginn EN, Agarwal R, Mills GB, Wasan $\mathrm{H}$ and Gabra H: DNA-PK mediates AKT activation and apoptosis inhibition in clinically acquired platinum resistance. Neoplasia 13: 1069-1080, 2011.

This work is licensed under a Creative Commons Attribution-NonCommercial-NoDerivatives 4.0 International (CC BY-NC-ND 4.0) License. 